\title{
Quality of Life and Associated Factors Among Family Caregivers of Adult Cancer Patients in Addis Ababa, Ethiopia
}

This article was published in the following Dove Press journal: Cancer Management and Research

\author{
Eyerusalem Yihedego $\mathbb{D}^{\prime}$ \\ Fekadu Aga $\mathbb{D}^{2}$ \\ Debela Gela (1D ${ }^{2}$ \\ Abdisa Boka $\mathbb{( D}^{2}$ \\ 'Oncology Nurse, Black Lion Specialized \\ Hospital, College of Health Science, \\ Addis Ababa University, Addis Ababa, \\ Ethiopia; ${ }^{2}$ School of Nursing \& Midwifery, \\ College of Health Science, Addis Ababa \\ University, Addis Ababa, Ethiopia
}

Correspondence: Debela Gela

School of Nursing \& Midwifery, College of Health Science, Addis Ababa University, P. O. Box: 44I2, Addis Ababa, Ethiopia

Tel +251 913179679

Email debegela@gmail.com
Background: Cancer has a major impact on the lives of family caregivers, including their health and quality of life (QOL). However, little is known about the QOL of family caregivers of adult cancer patients in Ethiopia. This study aimed to assess the QOL and associated factors among primary family caregivers of adult cancer patients in Addis Ababa, Ethiopia.

Methods: In this cross-sectional study, 291 family caregivers completed the survey in the Amharic language. The Caregiver Quality of Life Index-Cancer (CQOLC) was used to measure QOL of family caregivers. Descriptive and linear regression analyses were conducted using SPSS version 23.

Results: The mean age of the family caregivers was $37.04 \pm 11.47$ years and $51.5 \%$ were male. The mean score of QOL was $82.23( \pm 16.21)$. Not being employed in private sector $(\beta=$ -0.128 ; $\mathrm{CI}=-7.82,-0.45 ; p=0.028)$, having family monthly income less than 16 USD $(\beta=$ $0.132 ; \mathrm{CI}=0.87,10.88 ; p=0.021)$ and not having family monthly income greater than 64 USD $(\beta=-0.128 ; \mathrm{CI}=-10.43,-0.66 ; p=0.026)$, being spouse $(\beta=0.179 ; \mathrm{CI}: 1.34,11.99$; $p=0.019)$ and not residing in urban areas $(\beta=-0.139$; CI: $-10.53,-0.96 ; p=0.019)$ were negatively associated with the QOL of the family caregiver and explained $8.7 \%$ of the variation $\left(R^{2}=0.087 ; p=0.000\right)$.

Conclusion: Our findings identified factors such as occupation, income, relationship with the patient, and place of residence that negatively associated with the QOL of family caregivers. Targeted interventions such as social and economic support and bringing the care to the patient's residence place are needed to improve the QOL of family caregivers of adult cancer patients.

Keywords: quality of life, family caregiver, cancer, caregiver burden, Addis Ababa, Ethiopia

\section{Introduction}

Cancer has an enormous impact not only on the quality of life (QOL) of patients but also on their family caregivers. ${ }^{1-9}$ It has become an entire family's concern instead of being solely the problem of the individuals diagnosed with the disease and its chronicity has shifted care to the home, with family members being the core care providers. ${ }^{10,11}$ Family caregivers are those who provide not compensated care in the home and who have a pre-existing relationship to the individual for whom they provide care. ${ }^{4,12-15}$

Family caregivers are individuals - spouse, children, relative, or friend of the patient - who provide uncompensated physical care such as eating and moving 
around, and psychological, emotional, and social support to a family member with cancer, as well as communicate with the healthcare provider concerning changes in the patient's conditions and medications. ${ }^{16,17}$ They perform many activities including dealing with the diagnosis of cancer, managing treatment-related adverse events and disease-related symptoms, and tackling the challenges of end-of-life care. ${ }^{6}$ These often result in caregiver burden that usually leads to deterioration in QOL of the caregiver as caregiving requires much time and effort, great physical and emotional energy expenditure, and also hinders caregiver's social engagements. The role of caregiving can be highly stressful, burdensome, and subsequently, compromises the caregiver's QOL, which could lead to considerable impairment of physical, psychological, and social health. ${ }^{18,19}$

Caregiving for patients with cancer involves a multidimensional process and it requires intellectual and cognitive complexity and physical endurance. Family caregivers often assume the caregiving role with little or no preparation and without considering whether they have enough knowledge, resources, or skills. ${ }^{3,20,21}$ Studies have demonstrated that caregiving can positively affect patient's health outcomes and also have an impact on physical, mental, and social well-being of family caregivers of cancer patients. ${ }^{5,20}$

Quality of life of family caregivers is an issue of great interest in oncology because effective modern methods of cancer treatment and detection have led to an increase in the number of long-term survivors that can pose more burdens on the family caregivers. ${ }^{22}$ QOL is defined as individuals' perception of their life situation in the context of the culture and value systems in which they live and with their goals, expectations, standards, and concerns. It is a wide-ranging concept involving the person's physical health, psychological state, personal beliefs, social relationships and relationship to their environment. ${ }^{23,24}$

Ethiopia is one of the low-income sub-Saharan African countries. The taxable monthly employment income in Ethiopia ranges from 16 USD to over $295 \mathrm{USD}^{25}$ Since 2007, there was no census conducted in Ethiopia, this why it is difficult to have information on percentages of marital status, private and governmental employees of the population. The total number of populations of Ethiopia in 2017 was more than 105 million, of which more than 20 million was residing in urban areas. Out of urban found in Ethiopia, Addis Ababa is the capital city of the country with a total population of more than 2 million. $^{26}$
Caregiving for a sick relative is one of the most cherished family cultural values in this country. However, a study has shown that some sociocultural factors such as religious beliefs, economic issues, education, and social stigma and discrimination related to the nature of the disease may affect family caregiving in Ethiopia. ${ }^{27}$

Nevertheless, a decrease in the QOL of cancer family caregivers negatively influences the quality of care they provide to the patient. A multitude of factors may affect the QOL of family caregivers of adult cancer patients. These may include sociodemographic factors such as age, gender, religion, marital status, educational status, occupation, monthly income, types of relation to a patient, family size and place of residence $e^{2,3,5,7-9,12,28-30}$ and clinical characteristics of caregivers such as the presence of chronic disease ${ }^{8,9,19,31-34}$ Family caregiving experiences also vary from culture to culture which has enormous implications for the quality of life of family caregivers. For example, the cultural embeddedness of family caregiving imposes a sometimes burdensome responsibility on the caregivers, a condition that can negatively affect their quality of life in different sociocultural contexts. ${ }^{35,36}$ However, little is known about the QOL of family caregivers of adult cancer patients in the sociocultural context of Ethiopia though there are ubiquitous studies from high-income countries. Therefore, this study aimed to explore the QOL and associated factors among family caregivers of adult cancer patients in Addis Ababa, Ethiopia.

\section{Methods}

\section{Study Design, Setting, and Population}

This institutional-based cross-sectional study was conducted from March to May 2017 at Tikur Anbessa Hospital Oncology Center located in Addis Ababa, Ethiopia. Tikur Anbessa Hospital Oncology Center is the single largest institution for cancer treatment and cares in Ethiopia. All adults (18 years or older) primary family caregivers of cancer patients who were identified by patients as being a caregiver were included, except those who were critically ill at the time of data collection. The net sample of 291 was recruited using a systematic random sampling technique. This sample size yielded a statistical power of $99 \%$ with an assumed effect size of 0.3 and a significance level of 0.05 . The sampling frame for the systematic sampling technique was patients' follow-up registry excluding patients who were present for a first visit. The data were collected by five nurses holding 
a Bachelor of Science degree under the supervision of a senior nurse professional. The data collectors were provided two days of intensive training on the content of the measuring tool and participant recruitment strategies. The principal investigator also made a continuous follow-up to ensure the quality of the data collected. The research project was reviewed by an Institutional Review Board of the College of Health Sciences at Addis Ababa University. Permission to conduct the research was obtained from the authorities in the study setting and written informed consents were secured from each participant and the study was conducted in accordance with the Declaration of Helsinki.

\section{Measurements}

The sociodemographic characteristics of the participants were recorded using 10-items. These include age, gender, religion, marital status, educational level, monthly income, type of relationship with the patient, place of residence (urban/rural), family size, and presence of chronic disease. The Caregiver Quality of Life Index-Cancer (CQOLC) was used to measure QOL of family caregivers. The tool contains 35 items, which covers four domains: burden, disruptiveness, positive adaptation, and financial concerns. Participants were asked to give responses to each item on a 5-point Likert scale, from 0 (not at all) to 4 (very much). The total score (0-140) was obtained by summing up the scores of all items. Since higher score on CQOLC indicates increased caregiver burden, the high score indicates a low QOL. The scale had an internal consistency coefficient of 0.91 in the previous study. ${ }^{10}$ The internal consistency in this study was 0.88 which can be considered adequate. Data were collected in Amharic languages using an interviewer-administered questionnaire. In this regard, the questionnaire was translated from English to the Amharic language by a bilingual translator and then back-translated to English by another bilingual translator. Before data collection, we assessed the Amharic language version of the CQOLC to ensure meaning equivalence with the original English.

\section{Data Processing and Analysis}

Data were entered and cleaned using Epi-data version 3.1 and then transported to SPSS version 23.0 for analysis. Frequency distributions were computed for sociodemographic and clinical variables and mean values with standard deviations were calculated for QOL. The independent variables in this study were sociodemographic and clinical characteristics. The dependent variable was the QOL of the family caregiver. The sociodemographic variables such as age, gender, religion, marital status, educational status, occupation, monthly income, relation to the patient, family size, place of residence, and clinical variables such as the presence of chronic disease association with QOL were analyzed first by using a simple linear regression model. Then, only those variables with $\mathrm{p}$-values $<0.05$ were taken as a candidate for multiple linear regression analysis. In both simple and multiple linear regression models, p-value $<0.05$ was considered to be statistically significant.

\section{Results}

\section{Sociodemographic Characteristics of the Participants}

All of the study participants were interviewed, and this makes a $100 \%$ (291) response rate. All the participants were between age 18-70 with the mean age of 37.04 \pm 11.47 standard deviation years. The majority of the participants were $\leq 37$ years $(n=158,54.3 \%)$, male $(n=150$, $51.5 \%$ ), followers of Orthodox Christian church $(n=180,61.9 \%)$, married $(n=167,57.4 \%)$, college or university graduates $(\mathrm{n}=115,39.5 \%)$, private employees $(n=139,47.8)$, reside in urban areas $(n=236,81.1 \%)$, had family size of $2-6(n=187,64.3 \%)$, earn a monthly income between 16 and 32 USD ( $n=69,23.7 \%)$, and did not have chronic disease $(\mathrm{n}=148,50.9 \%)$ such as hypertension, diabetes, arthritis, cardiovascular disorders, TB, asthma and HIV/AIDS (Table 1).

\section{Quality of Life of Participants}

The overall mean score of the QOL of the participants was $82.23( \pm 16.21)$. The subscale scores for burden was 24.49 $( \pm 7.83)$, for disruptiveness was $16.63( \pm 5.69)$, for positive adaptation was $18.58( \pm 3.42)$, for financial concern was $9.29( \pm 3.27)$, and for other subscale scores was 12.94 $( \pm 4.18)$ (Table 2).

\section{Factors Associated with Quality of Life}

In simple linear regression, we found that being employed in the private sector $(p=0.017)$, having family monthly income less than 16 USD $(p=0.007)$ and greater than 64 USD ( $p=0.019)$, being the spouse $(p=0.045)$ and residing in urban areas $(p=0.007)$ were significantly associated with the QoL (CQOLC) of family caregivers.

A standard multiple linear regression was performed to measure the factors that associated with the QoL of family 
Table I Socio-Demographic and Clinical Characteristics of Study Participants by CQOLC Score (N= 29I)

\begin{tabular}{|c|c|c|c|}
\hline \multicolumn{2}{|l|}{ Characteristics } & \multirow{2}{*}{$\begin{array}{l}\text { n (\%) } \\
158(54.3)\end{array}$} & \multirow{3}{*}{$\begin{array}{l}\text { CQOLC Score, Mean (SD) } \\
82.6(16.60) \\
81.4(15.54)\end{array}$} \\
\hline Age (in years) & $\leq 37$ & & \\
\hline Mean $(S D)=37.04 \pm 1 \mathrm{I} .47$ & $>37$ & $133(45.7)$ & \\
\hline \multirow[t]{2}{*}{ Gender } & Male & $\mid 50(5 \mid .5)$ & $83.4(\mid 5.5 I)$ \\
\hline & Female & $|4|(48.5)$ & $80.5(16.65)$ \\
\hline \multirow[t]{3}{*}{ Religion } & Orthodox & $180(61.9)$ & $83.4(16.44)$ \\
\hline & Muslim & $55(18.9)$ & $81.9(14.18)$ \\
\hline & Protestant & $56(19.2)$ & $78.6(17.02)$ \\
\hline \multirow[t]{4}{*}{ Marital status } & Single & $100(34.4)$ & $82.5(17.76)$ \\
\hline & Married & $167(57.4)$ & $81.7(14.92)$ \\
\hline & Divorced & $19(6.5)$ & $86.3(|3.2|)$ \\
\hline & Widowed & $5(1.7)$ & $66.0(23.82)$ \\
\hline \multirow[t]{4}{*}{ Education status } & Cannot read and write & $3 I(10.7)$ & $83.9(\mid 4.45)$ \\
\hline & Primary & $44(15.1)$ & $84.7(13.69)$ \\
\hline & Secondary & $101(34.7)$ & $82.4(15.78)$ \\
\hline & College or university graduated & $115(39.5)$ & $80.1(17.56)$ \\
\hline \multirow[t]{7}{*}{ Occupation } & House wife & $25(8.6)$ & $84.0(14.49)$ \\
\hline & Government employee & $69(23.7)$ & $84.5(\mid 4.40)$ \\
\hline & Private employee & $139(47.8)$ & $79.6(17.46)$ \\
\hline & Farmer & $30(10.3)$ & $84.3(13.85)$ \\
\hline & Jobless & $12(4.1)$ & $82.8(16.78)$ \\
\hline & Student & $12(4.1)$ & $84.8(17.24)$ \\
\hline & Pension & $4(1.4)$ & $83.5(\mid 2.87)$ \\
\hline \multirow[t]{5}{*}{ Family income } & $<16$ USD & $46(15.8)$ & $88.0(13.92)$ \\
\hline & 16-32 USD & $69(23.7)$ & $82.4(16.70)$ \\
\hline & 32-48 USD & $62(21.3)$ & $83.9(\mid 6.8 I)$ \\
\hline & 48-64 USD & $65(22.3)$ & $79.1(14.88)$ \\
\hline & $>64$ USD & $49(16.8)$ & $77.3(16.2 I)$ \\
\hline \multirow[t]{4}{*}{ Relation to patient } & Spouse & $74(25.4)$ & $84.3(15.57)$ \\
\hline & Child & $93(32.0)$ & $81.8(16.42)$ \\
\hline & Parent & $40(13.7)$ & $82.2(14.43)$ \\
\hline & Other* & $84(28.9)$ & $80.1(16.97)$ \\
\hline \multirow[t]{2}{*}{ Place of residence } & Urban & $236(81.1)$ & $80.8(\mid 5.5 I)$ \\
\hline & Rural & $55(18.9)$ & $87.1(17.72)$ \\
\hline \multirow[t]{3}{*}{ Family size } & $2-6$ & $187(64.3)$ & $81.4(16.25)$ \\
\hline & $7-11$ & $96(33.0)$ & $83.8(16.14)$ \\
\hline & $12-16$ & $8(2.7)$ & $75.8(9.79)$ \\
\hline \multirow[t]{2}{*}{ Presence of chronic disease** } & No & $148(50.9)$ & $81.5(16.48)$ \\
\hline & Yes & $143(49.1)$ & $82.5(15.75)$ \\
\hline
\end{tabular}

Notes: *Relative friend; **Hypertension, Diabetes, Arthritis, Cardiovascular disorders, TB, Asthma, HIV/AIDS.

Abbreviation: USD, United States Dollar.

caregivers. The independent variables entered in the analysis were having private sector occupation, having family monthly income less than 16 USD and greater than 64 USD, being the spouse, and residing in urban areas with the total score on the CQOLC as dependent variable. Not being employed in private sector $(\beta=-0.128 ; \mathrm{CI}=-7.82,-0.45 ; p=$ $0.028)$, having family monthly income less than 16 USD ( $\beta=$ $0.132 ; \mathrm{CI}=0.87,10.88 ; p=0.021)$ and not having family monthly income greater than 64 USD $(\beta=-0.128$; $\mathrm{CI}=$ $-10.43,-0.66 ; p=0.026)$, being spouse $(\beta=0.179$; CI: 
Table 2 Quality of Life of the Family Caregivers: Mean, Standard Deviation, Maximal Score, and Cronbach's Alpha of Subscales $\operatorname{CQOLC}(n=291)$

\begin{tabular}{|l|l|l|l|}
\hline Subscales & $\begin{array}{l}\text { Maximum } \\
\text { Score }\end{array}$ & Mean( \pm SD) & $\alpha^{*}$ \\
\hline Burden (10 items) & 40 & $24.49( \pm 7.83)$ & 0.79 \\
$\begin{array}{l}\text { Disruptiveness (7 items) } \\
\text { Positive adaptation (7 }\end{array}$ & 28 & $16.63( \pm 5.69)$ & 0.70 \\
items) & $18.58( \pm 3.42)$ & 0.71 \\
Financial concern (3 & 12 & $9.29( \pm 3.27)$ & 0.81 \\
items) & 32 & $12.94( \pm 4.18)$ & 0.74 \\
Other** (8 items) & 140 & $82.23( \pm 16.21)$ & 0.88 \\
Total score of QOL (35 & & & \\
items) & & & \\
\hline
\end{tabular}

Notes: *Cronbach's alpha. **Item without categories.

Abbreviation: CQOLC, Caregivers Quality Of Life Index-Cancer.

$1.34,11.99 ; p=0.019)$ and not residing in urban areas $(\beta=$ -0.139 ; CI: $-10.53,-0.96 ; p=0.019)$ were negatively associated with the QOL of the family caregiver and explained $8.7 \%$ of the variation $\left(R^{2}=0.087 ; p=0.000\right)$ (Table 3 ).

\section{Discussion}

In this study, we explored the QOL and associated factors among family caregivers of adult cancer patients in Addis Ababa, Ethiopia. The mean age of the study participants was $37.04 \pm 11.47$ standard deviation years. There was a statistically significant positive association between QOL and family monthly income (income greater than 64 USD) and residing in urban areas, but there was a statistically significant negative association between QOL and occupation (being employed in the private sector), family monthly income (income less than 16 USD) and relationship with the patients (being spouse).

Our study identified that occupation; monthly income; spousal relationship with the patients; and residing in urban areas have an association with QOL of family caregivers of adult cancer patients. This study revealed that cancer family caregivers who were private sector had low QOL compared with those who had other occupations (government employees, farmer, jobless, student). Although per our search there is no study done on this variable, this might be related to the fact that cancer family caregivers who employed in the private sector have time constraints due to lack of freedom and prolonged working time when compared to the other occupations. Thus, family caregivers of adult cancer patients who were employed in the private sector require close attention and care to improve their QOL.

This study shows that cancer family caregivers who had monthly income greater than 64 USD had high QOL compared with those who had lower monthly income. This finding is similar to other studies done in other settings that found family caregivers with a lower monthly income had low QOL. 5,7,8,19,28,34,37 This implies that family caregivers with lower monthly income may have a greater economic (financial) burden to deal with the cost of treatments and access to the health facilities. Therefore, cancer family caregivers with lower monthly income need special emphasis when designing and implementing interventions aimed at improving the QOL of this population group. This may involve the provision of economic support through established safety net programs in the community.

Our study shows that family caregivers who had a spousal relationship with the patients have low QOL compared with those who had other relationships (child, parent, relative, friend). This corroborates with the findings of studies from other settings that found spousal relationship has the strongest impact on family caregivers' QOL. 9,12,20,34 This might be related to the fact that spouses shoulder most of the caregiving responsibility but are less likely than other family caregivers to receive assistance. Thus, it is necessary to design a mechanism in which spousal family caregivers

Table 3 Factors Associated with Quality of Life of Cancer Patients' Caregivers

\begin{tabular}{|l|l|l|l|l|l|l|}
\hline \multirow{2}{*}{ Independent Variables } & Category & \multicolumn{2}{l|}{ Simple Linear Regression } & \multicolumn{3}{l|}{ Multiple Linear Regression } \\
\cline { 3 - 7 } & & $\boldsymbol{B}$ & $\boldsymbol{p}$-value & $\boldsymbol{\beta}$ & $\mathbf{9 5 \%} \mathbf{C l}$ & $\boldsymbol{p}$-value \\
\hline Occupation & Private employee & -0.134 & 0.017 & -0.128 & $-7.82,-0.45$ & $0.028^{*}$ \\
Family income & $<16$ USD & 0.159 & 0.007 & 0.132 & $0.87,10.88$ & $0.02 I^{*}$ \\
Family income & $>64$ USD & -0.137 & 0.019 & -0.128 & $-10.43,-0.66$ & $0.026^{*}$ \\
Relation to patient & Spouse & 0.090 & 0.045 & 0.179 & $1.34,11.99$ & $0.014^{*}$ \\
Place of residence & Urban & -0.157 & 0.007 & -0.139 & $-10.53,-0.96$ & $0.019^{*}$ \\
\hline
\end{tabular}

Notes: Standard multiple regression analysis of family caregiver's quality of life (dependent variable) and demographics (independent variables; $\mathrm{n}=29 \mathrm{I}$ ). Dependent variable

$=$ total score of quality of life (CQOLC). $\mathrm{R}$ square $=0.087$; SE estimate $=15.62 .{ }^{*} \mathrm{p}<0.05$

Abbreviations: CQOLC, Caregivers Quality Of Life Index-Cancer; USD, United States Dollar. 
get some form of social support to alleviate the caregiving burden they experience and improve their QOL.

This study also shows that family caregivers who were residing in urban areas had high QOL compared to those who were from rural areas. Though limited, previous studies have also shown that residing in urban areas are positively associated with QOL of family caregivers of adult cancer patients. ${ }^{29,30,38}$ This might be related to the fact that residing in rural areas are often associated with delays in diagnosis, challenges of traveling long distances to urban centers, and limited access to oncology services that pose a burden on the family caregivers and affect their QOL. Therefore, giving special attention to the needs of family caregivers residing in rural areas is essential. This be done by bringing care and support nearer to their dwelling places through the implementation of community-based chronic illness care model. ${ }^{39}$

Our study did not find an association between age, gender, religion, family size, and presence of chronic disease and QOL. This is contrary to the findings of studies conducted in other settings that found age, gender, religion, family size and presence of chronic disease had an association with the QOL of cancer family caregivers. ${ }^{2,3,5,9,11,28,34}$ The discrepancy may be linked to the difference in the measurement tools used. While CQOLC was used in the present study, others have used tools such as FS-12 and Zarit Burden Inventory for measuring the QOL of cancer family caregivers.

\section{Strengths and Limitations}

This study has a couple of strengths. One, it is the first study on cancer family caregivers' QOL in Ethiopia. Secondly, the study had a $100 \%$ response rate. Our study also has some limitations. Firstly, the use of a crosssectional design does not allow inferring causality or having a control group. Prospective and experimental studies are warranted. Secondly, the use of an interviewer-administered structured questionnaire for data collection. Using this method to identify QOL and associated factors among family caregivers of adult cancer patients might involve some risk of information concealing. Thus, future research should incorporate qualitative interviews since it lets participants to liberally highlight the impact of caregiving on their QOL. Finally, some potential influencing factors such as family hardiness, coping, and social supports were not measured in this study.

\section{Conclusion}

This study showed that not being employed in the private sector, having a lower monthly income, spousal relationship with cancer patients, and residing in rural areas were the factors that associated with poor QOL of family caregivers of adult cancer patients. This means having a higher income can positively associated with the QOL of family caregivers. Therefore, future interventions should focus on establishing a social safety net program that can provide economic support for low-income family caregivers and social support for those in spousal relationships. Further research on the association of QOL of family caregivers of adult cancer patients and family hardiness, coping, and social support are warranted.

\section{Abbreviations}

AOR, adjusted odds ratio; CI, confidence interval; CQOLC, Quality Of Life Index-Cancer; HIV/AIDS, human immunodeficiency virus/acquired immune deficiency syndrome; QOL, quality of life; SPSS, Statistical Package for the Social Sciences; TB, tuberculosis; USD, United States Dollar.

\section{Data Sharing Statement}

Datasets used and/or analyzed during the current study are available from the corresponding author on reasonable request.

\section{Ethics Approval and Consent to Participate}

This study was reviewed and approved by an Institutional Review Board of the College of Health Sciences, Addis Ababa University. All subjects provided written informed consent, and the study was conducted in accordance with the Declaration of Helsinki.

\section{Acknowledgment}

We are grateful to Addis Ababa University for the provision of financial support to this research project.

\section{Author Contributions}

All authors made substantial contributions to conception and design, acquisition of data, or analysis and interpretation of data; took part in drafting the article or revising it critically for important intellectual content; agreed to submit to the current journal; gave final approval of the version to be published; and agree to be accountable for all aspects of the work. 


\section{Funding}

The study was funded by Addis Ababa University.

\section{Disclosure}

The authors declare that they have no competing interests.

\section{References}

1. Kim Y, Given B. Quality of life of family caregivers of cancer survivors: across the trajectory of the illness. Cancer. 2008;112(11 Suppl):2556-2568. doi:10.1002/cncr.23449

2. Li Q, Xu Y, Zhou H, Loke AY. Factors influencing the health-related quality of life of Chinese advanced cancer patients and their spousal caregivers: a cross-sectional study. BMC Palliat Care. 2016;15:72. doi:10.1186/s12904-016-0142-3

3. Meecharoen W, Sirapo-ngam Y, Monkong S, Oratai P, Northouse LL. Factors influencing quality of life among family caregivers of patients with advanced cancer: a causal model. Pacific Rim Int $J$ Nurs Res. 2013;17(4):304-316.

4. Ferrell B, Hanson J, Grant M. An overview and evaluation of the oncology family caregiver project: improving quality of life and quality of care for oncology family caregivers. Psychooncology. 2013;22(7):1645-1652. doi:10.1002/pon.3198

5. Choi YS, Hwang SW, Hwang IC, et al. Factors associated with quality of life among family caregivers of terminally ill cancer patients. Psycho-Oncology. 2016;25:217-224. doi:10.1002/pon.3904

6. Chindaprasirt J, Limpawattana P, Pakkaratho P, et al. Burdens among caregivers of older adults with advanced cancer and risk factors. Asian Pac J Cancer Prev. 2014;15(4):1643-1648. doi:10.7314/ APJCP.2014.15.4.1643

7. Zhu P, Fu J-F, Wang B, et al. Quality of life of male spouse caregivers for breast cancer patients in China. Asian Pac J Cancer Prev. 2014;15(10):4181-4185.

8. Yun YH, Rhee YS, Kang IO, et al. Economic burdens and quality of life of family caregivers of cancer patients. Oncology. 2005;68(2-3):107-114. doi:10.1159/000085703

9. Lu L, Pan B, Sun W, Cheng L, Chi T, Wang L. Quality of life and related factors among cancer caregivers in China. Psychiatry Clin Neurosci. 2010;64(5):505-513. doi:10.1111/j.1440-1819.2010.02131.x

10. Weitzner MA, Jacobsen PB, Wagner H Jr., Friedland J, Cox C. The Caregiver Quality of Life Index-Cancer (CQOLC) scale: development and validation of an instrument to measure quality of life of the family caregiver of patients with cancer. Qual Life Res. 1999;8 (1-2):55-63.

11. Lim HA, Tan JY, Chua J, et al. Quality of life of family caregivers of cancer patients in Singapore and globally. Singapore Med J. 2017;58 (5):258-261. doi:10.11622/smedj.2016083

12. Kim Y, Baker F, Spillers RL. Cancer caregivers' quality of life: effects of gender, relationship, and appraisal. J Pain Symptom Manage. 2007;34(3):294-304. doi:10.1016/j.jpainsymman.2006.11.012

13. Musich S, Wang SS, Kraemer S, Hawkins K, Wicker E. Caregivers for older adults: prevalence, characteristics, and health care utilization and expenditures. Geriatr Nurs. 2017;38(1):9-16. doi:10.1016/j. gerinurse.2016.06.017

14. Bevans MF, Sternberg EM. Caregiving burden, stress, and health effects among family caregivers of adult cancer patients. JAMA 2012;307(4):398-403. doi:10.1001/jama.2012.29

15. Akpan-Idiok PA, Anarado AN. Perceptions of burden of caregiving by informal caregivers of cancer patients attending University of Calabar Teaching Hospital, Calabar, Nigeria. Pan Afr Med J. 2014;18.

16. Ashrafian S, Feizollahzadeh H, Rahmani A, Davoodi A. The unmet needs of the family caregivers of patients with cancer visiting a referral Hospital in Iran. Asia-Pac J Oncol Nurs. 2018;5 (3):342-352. doi:10.4103/apjon.apjon_7_18
17. Effendy C, Vernooij-Dassen M, Setiyarini S, et al. Family caregivers' involvement in caring for a hospitalized patient with cancer and their quality of life in a country with strong family bonds. Psychooncology. 2015;24:585-591. doi:10.1002/pon.3701

18. Stajduhar KI. Burdens of family caregiving at the end of life. Clin Invest Med. 2013;36:3. doi:10.25011/cim.v36i3.19722

19. Yang X, Hao Y, George SM, Wang L. Factors associated with health-related quality of life among Chinese caregivers of the older adults living in the community: a cross-sectional study. Health Qual Life Outcomes. 2012;10:143. doi:10.1186/1477-7525-10-143

20. Northouse L, Williams A-L, Given B, McCorkle R. Psychosocial care for family caregivers of patients with cancer. J Clin Oncol. 2012;30(11):1227-1234. doi:10.1200/JCO.2011.39.5798

21. Northouse LL, Katapodi MC, Song L, Zhang L, Mood DW. Interventions with family caregivers of cancer patients: meta-analysis of randomized trials. CA Cancer J Clin. 2010;60(5):317-339.

22. Awadalla AW, Ohaeri JU, Gholoum A, Khalid AOA, Hamad HMA, Jacob A. Factors associated with quality of life of outpatients with breast cancer and gynecologic cancers and their family caregivers: a controlled study. BMC Cancer. 2007;7(1):102. doi:10.1186/14712407-7-102

23. GroupWhoqol. Development of the WHOQOL: rationale and Current Status. Int J Ment Health. 1994;23(3):24-56. doi:10.1080/00207 411.1994.11449286

24. Lavdaniti M, Tsitsis N. Definitions and conceptual models of quality of life in cancer patients. Health Sci J. 2015;9(2-6):1-5.

25. The Federal Democratic Republic of Ethiopia (FDRE). Federal Income Tax Proclamation 2016. Proclamation No. 979/2016. Federal Negarit Gazette, 22nd Year, No.104. 2016.

26. Worldometer. Ethiopia Population; 2017. Available from: https:// www.worldometers.info/world-population/ethiopia-population/. Accessed August 08, 2020.

27. Aga F, Kylma J, Nikkonen M. Sociocultural factors influencing HIV/ AIDS caregiving in Addis Ababa, Ethiopia. Nurs Health Sci. 2009;11:244-251. doi:10.1111/j.1442-2018.2009.00448.x

28. Kim Y, Spillers RL. Quality of life of family caregivers at 2 years after a relative's cancer diagnosis. Psycho-Oncology. 2010;19 (4):431-440. doi:10.1002/pon.1576

29. Loughery J, Woodgate RL. Supportive care needs of rural individuals living with cancer: a literature review. Can Oncol Nurs J. 2015;25 (2):157-166. doi: $10.5737 / 23688076252157166$

30. Thirumoorthy A, Devi PS, Thennarusu K. Quality of life among the caregivers of persons living with cancer. IOSR J Humanities Soc Sci. 2016;21(2):23-30.

31. Rochaa RS, Pinheirob LP, Oriáa MOB, Ximenesa LB, Pinheiroa AKB, Aquinoa P. Social determinants of health and quality of life of caregivers of children with cancer. Rev Gaúcha Enferm. 2016;37:3.

32. Palos GR, Mendoza TR, Liao KP, et al. Caregiver symptom burden: the risk of caring for an underserved patient with advanced cancer. Cancer. 2011;117(5):1070-1079. doi:10.1002/cncr.25695

33. Yoon H Quality of Life and Depression of Korean American Cancer Patients and Their Family Caregivers.:2015.

34. Hacialioglu N, Ozer N, Karabulutlu EY, Ne E, Erci B. The quality of life of family caregivers of cancer patients in the East of Turkey. Eur J Oncol Nurs. 2010;14:211-217. doi:10.1016/j.ejon.2010.01.017

35. Pharr JR, Dodge Francis C, Terry C, Clark MC. Culture, caregiving, and health: exploring the influence of culture on family caregiver experiences. ISRN Public Health. 2014;2014:1-8. doi:10.1155/2014/ 689826

36. Githaiga JN, Swartz L. Socio-cultural contexts of end- of- life conversations and decisions: bereaved family cancer caregivers' retrospective co-constructions. BMC Palliat Care. 2017;16(1):40. doi:10.1186/s12904-017-0222-z

37. Nayak MG, George A, Vidyasagar MS, Kamath A. Quality of life of family caregivers of patients with advanced cancer. IOSR $J$ Nurs Health Sci. 2014;3(2):70-75. doi:10.9790/1959-03217075 
38. Brazil K, Kaasalainen S, Williams A, Dumont SA. Comparison of Support Needs Between Rural and Urban Family Caregivers Providing Palliative Care. Am J Hospice Palliative Med. 2014;31 (1):13-19. doi:10.1177/1049909112474712
39. Davy C, Bleasel J, Liu H, Tchan M, Ponniah S, Brown A. Effectiveness of chronic care models: opportunities for improving healthcare practice and health outcomes: a systematic review. $B M C$ Health Serv Res. 2015;15:194. doi:10.1186/s12913-015-0854-8

\section{Publish your work in this journal}

Cancer Management and Research is an international, peer-reviewed open access journal focusing on cancer research and the optimal use of preventative and integrated treatment interventions to achieve improved outcomes, enhanced survival and quality of life for the cancer patient.
The manuscript management system is completely online and includes a very quick and fair peer-review system, which is all easy to use. Visit http://www.dovepress.com/testimonials.php to read real quotes from published authors. 